\title{
Size Independent Active Contour Model for Blood Vessel Lumen Quantification in High-Resolution Magnetic Resonance Images
}

\author{
Catherine Desbleds-Mansard ${ }^{1}$, Alfred Anwander ${ }^{1}$, Linda Chaabane ${ }^{2}$, \\ Maciej Orkisz ${ }^{1}$, Bruno Neyran ${ }^{1}$, Philippe C. Douek ${ }^{1}$, Isabelle E. Magnin ${ }^{1}$ \\ ${ }^{1}$ CREATIS, CNRS Research Unit (UMR 5515) affiliated to INSERM, Lyon, France \\ ${ }^{2}$ Laboratory RMN, CNRS Research Unit (UMR 5012), Lyon, France \\ Correspondence address: CREATIS, INSA de Lyon, bât. Blaise Pascal, \\ 7 rue J. Capelle, 69621 Villeurbanne cedex, France \\ catherine.mansard@creatis.insa-lyon.fr
}

\begin{abstract}
Atherosclerosis is the most common cause of myocardial infarction. To study the atherosclerotic plaque in high resolution Magnetic Resonance images we developed a software tool called ATHER. An active contour model is used for segmentation and quantification of blood vessel lumen. Its implementation, based on a dynamic scaling process, presents two interesting features: 1) independence of the influence of the inflating force from the current size of the contour, 2) strong reduction of the computational cost. Therefore the contour converges very quickly even when initialized by a single point. This paper reports a validation of the model in ex vivo vascular images from Watanabe heritable hyperlipidaemic rabbits. Results of automatic quantification were compared to measurements performed by experts. Average difference of the area measurements between ATHER and the experts was equal to the interobserver variability, but intra-variability of the automatic measurements was significantly smaller than the intra-observer variability.
\end{abstract}

\section{Introduction}

Atherosclerosis is the most common cause of myocardial infarction and stroke. To prevent these acute accidents due to severe lumen obstruction and/or to the atherosclerotic plaque rupture, there is a growing number of investigations dealing with the plaque identification, evolution and characterization both in humans and in animal models [1-5]. In particular, Watanabe heritable hyperlipidaemic (WHHL) rabbit was described as a well suited model for the atherosclerosis. The studies of the plaque evolution require quantification of the vascular lumen and of the plaque in numerous images. This tedious task needs to be computer-assisted [6]. To do this, one can use a general-purpose software such as Osiris (University Hospital Geneva Switzerland). However, a specific application-tailored software is expected to provide better ergonomics and more precise results. The objective of the hereafter presented work was to realize such a software tool with a user-friendly graphical interface. It will be hereafter referred to as ATHER. 
The quantification of the vascular lumen is based on an automatic extraction of its boundary. It is well known that simple edge detection often leads to discontinuous lines with gaps and protrusions, because of noise and of contrast variations, especially in the medical images context. In this context, techniques based on minimum-cost path search [7], on deformable models [8] or on deformable templates [9] are usually applied to extract smooth closed boundaries as expected in most cases, in particular in the case of the vascular lumen. A deformable model (curve, surface ...) is defined by the expected shape properties, by some "mechanical" properties (flexibility, elasticity) and by forces which gradually deform it to make it coincide with the actual boundaries in the image. External forces attract the initial form towards the points likely to belong to a boundary (e.g. maximum of the gradient), while internal forces attract it towards the expected reference shape. Many improvements of the very first implementation of planar deformable models, named active contours or snakes [10], were proposed in order to make the result initialization-independent.

Our original implementation, called Dynamic Active Contour (DAC), needs a single initialization point inside the lumen [11] and can propagate on a whole sequence of images representing contiguous vascular cross-sections. This paper describes a validation of its application to vascular lumen quantification in ex vivo high resolution magnetic resonance (MR) images from WHHL rabbits. Preliminary results of this validation were presented in [12]. The sequel of the paper will be organized as follows: short description of the animals and of the image acquisition protocol, presentation of the DAC model, summary of the characteristics of the graphical interface, explanation of the validation method and discussion of the obtained results.

\section{Animals and Images}

Six WHHL rabbits have been included in the entire study: three homozygous and three heterozygous, two of which had a lipid-rich diet. First, two kinds of in vivo MR images were acquired: one set of images focused on the vascular wall, the other one used a contrast agent in order to highlight the vessel lumen. Then the animals were sacrificed at age between 13 and 18 months. The entire aorta, heart and kidneys were removed after fixation under perfusion with paraformaldehyde. In vitro images of arteries thus obtained were used for the purpose of our validation.

The in vitro high resolution MR imaging was performed with a $2 \mathrm{~T}$ horizontal Oxford magnet and a SMIS console. The specimens were placed in a half-birdcage RF coil of $25 \mathrm{~mm}$ diameter working at $85.13 \mathrm{MHz}$. High resolution axial images of the thoracic and abdominal aorta were taken using a multi-slice 2D spin-echo sequence with a $256 \times 128$ matrix. The slice thickness was $1-0.8 \mathrm{~mm}$ and the pixel size was from 58 to $78 \mu \mathrm{m}$. $\mathrm{T}_{1}$ and $\mathrm{T}_{2}$ weighted images were obtained with $\mathrm{TR} / \mathrm{TE}=600 / 21 \mathrm{~ms}$ and $1800 / 50 \mathrm{~ms}$ respectively. Six territories of exploration have been studied from the aortic arch down to the iliac bifurcation. The acquired images were organized into series of contiguous slices, one series per territory. 


\section{Dynamic Active Contour Model}

A planar closed active contour (snake) is approximated by a set of points defined in terms of the $x$ and $y$ coordinates, which are parameterized by $i$, a curvilinear parameter, and $k$ a time parameter:

$$
\mathbf{v}(i, k)=(x(i, k), y(i, k))^{T} .
$$

These points are vertices of a polygon which alters its shape and size in an iterative process while seeking the state corresponding to an energy minimum. The energy of the snake is made up of internal and external energy terms :

$$
E(\mathbf{v})=E_{\text {int }}(\mathbf{v})+E_{\text {ext }}(\mathbf{v}) \text {. }
$$

The internal energy depends solely on the shape of the contour and controls the elasticity and flexibility of the snake. It is computed from the first and second derivatives of the curve defined by the vertices. This energy prevents irregular deformations of the contour. The external energy depends on the image intensity values on the vertices. A balloon pressure [13] is added to the external energy so as to inflate the contour outwards. Differential calculus minimizes the two energy terms and aligns the regularized snake with boundaries in the image. The deformation in the plane is done in discrete time steps according to the following evolution equation:

$$
\mathbf{v}(i, k)=[\gamma \mathbf{I}+\mathbf{A}]^{-1}\left[\gamma \mathbf{v}(i,(k-1))+F_{\text {ext }}(\mathbf{v}(i,(k-1)))\right] .
$$

The matrix A represents a discretized formulation of the internal energy. The derivatives of the contour are computed by finite differences using a curvilinear sampling distance $h$. The damping parameter $\gamma$ controls the snake deformation magnitude per iteration.

In the conventional active contour implementation, after deformation, the active contour changes its size and shape, and the initial discretization step $h$ does not correspond to the real distance between the snake points. Numerical computations of the derivatives of the contour are sensitive to the sampling distance and the evolution equation is only valid if the snake points are equally distanced and the step $h$ is unchanged. If the actual distance between the points differs from the initial step $h$, the internal energy is mis-evaluated and the growth of the snake stops before the boundary is reached. Hence, in the usual implementations, the snake has to be resampled and a new model with a different sampling distance and a different number of snake points has to be set. This requires the inversion of the system matrix $[\gamma \mathbf{I}+\mathbf{A}]^{-1}$, which is a time-consuming task.

In our Dynamic Active Contour model, instead of the rediscretization of the model, the actual size of the contour is scaled to a reference size. The model is set once with a reference size fixed and with a contour length equal to the number of points (sampling distance $h=1$ ). The same model is used at each iteration step. The actual snake is scaled to this normalized size:

$$
\mathbf{v}^{\prime}(i,(k-1))=\mathbf{v}(i,(k-1)) / h_{k-1},
$$


where $h_{k-1}$ is the sampling distance of the actual contour. This only modifies the coordinates of the snake points, but each point keeps the external-force vector calculated before the scaling. The reference model is used to compute the regularized deformation vector according to this external force vector. This deformation vector

$$
\Delta \mathbf{v}^{\prime}(i, k)=\mathbf{v}^{\prime}(i, k)-\mathbf{v}^{\prime}(i,(k-1))
$$

is directly applied on the non-scaled snake to compute the new contour:

$$
\mathbf{v}(i, k)=\mathbf{v}(i,(k-1))+\Delta \mathbf{v}^{\prime}(i, k) .
$$

Since the deformation modifies the snake's shape, the distances between the vertices are unequal. The fixed number of snake points is redistributed on the polygonal contour. The perimeter of the polygon is computed and divided by the number of points. This new distribution step is applied to the polygon by sampling the outline at fixed distances $h_{k}$ and by defining new vertices for the next iteration. The deformation is scale-independent, and the final outline is independent from the initialization. The dynamic scaling allows an initialization with a small circle of the size of only one pixel. Regular sampling distributes a sufficient number of $N$ snakepoints on the circle. The number $N$ is about the length of the final contour.

The deformation speed of the dynamic active contour does not change with the size of the contour and can be fixed to a speed of one pixel per iteration by the damping parameter $\gamma$. The scaling and redistribution of the snake-points are computationally very fast and simple tasks and do not slow down the snake deformation speed. The snake does not need to be reinitialized with a varying number of points. This is a big advantage compared to traditional snake implementations which need a setup of the system matrix at each iteration.

\section{Graphical Interface : ATHER}

ATHER is our custom software for atherosclerosis MR image visualization and quantification. It displays one entire image series at a time. Each slice can be zoomed, its histogram can be interactively stretched and signal to noise ratio as well as the standard deviation of noise can be measured in user-selected regions. Vessel lumen contours can both be manually drawn and automatically extracted, with a possibility of interactive refinement if necessary. The automatic contour extraction needs no parameter tuning, since the parameters of the DAC model have been experimentally optimized for this application. Hence, the only necessary user interaction is a doubleclick in the vessel lumen in the selected slice. For further speed-up, the contour extraction can be performed in the entire series. The extracted contour's gravity center become the starting point for the contour extraction in the next slice. This method is applicable because each series represents a short, approximately straight, portion of the aorta (fig. 1a). Quantitative results (area, perimeter and diameter) are displayed and stored in a computer file. ATHER also features such additional functionalities as automatic extraction of the external contour of the vessel wall, as well as semiautomatic plaque quantification (fig 1b). They will not be detailed in this paper, because their validation is still ongoing. 
(a)

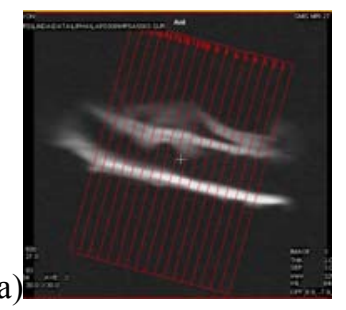

(b)
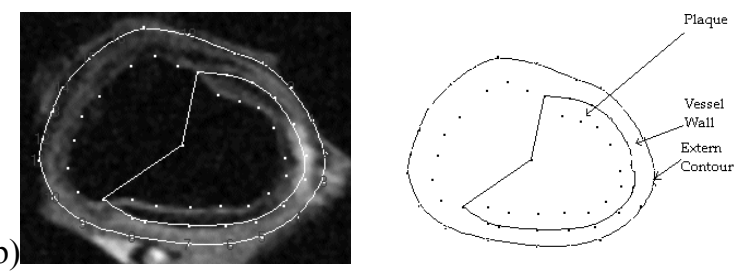

Fig. 1. Longitudinal section of a rabbit artery with lines showing the positions of cross sections from the corresponding series (a). Cross-section with internal and external contours of the vessel wall superimposed, and plaque delineated (b)

\section{$5 \quad$ Measurement Protocol}

Images from a subset of three amongst six rabbits were used for the purpose of validation of the DAC model. For these rabbits, expert-done measurements of the lumen area were already available for 121 slices. Indeed, these contours have been traced six months earlier, by an expert using Osiris software (University Hospital Geneva Swizterland). Let us note that Osiris also offers the possibility of an automatic contour extraction. However, it needs to be done in each slice separately. In practice, the expert used this automatic extraction and stored the result when, in her opinion, the contour perfectly fit the boundary. Otherwise she drew it manually. Only the area measurements have been stored, while the contour points themselves were lost.

Therefore, the first quantitative validation was based on area measurements. The areas of the contours traced by the expert were compared with the areas of the contours automatically extracted by ATHER in all the 121 slices. Average absolute difference was calculated as:

$$
\mu=\operatorname{mean}_{i}\left(\frac{\left|\operatorname{Area}(i)_{\text {ATHER }}-\operatorname{Area}(i)_{\text {EXPERT }}\right|}{\operatorname{Area}(i)_{\text {EXPERT }}}\right) * 100 .
$$

ATHER's variability was calculated according to a similar formula, after applying the automatic contour extraction twice to all the data set. The users were authorized to change the initialization point in single image within a series but not to modify the resulting contours. Then, in order to quantify the operator-dependence of the nonautomatic measurements, the same expert re-traced the contours in 45 slices ( 5 series) and a second expert also traced them twice in the same 45 slices. For the first expert the time interval between two measurements was six months, while for the second one it was one month and half.

For a qualitative comparison between ATHER and the experts, contours' forms were visually inspected. However, area measurements combined with qualitative appreciation might appear relatively forgiving. Hence the points of the contours traced by the second expert were stored and distances between these contours and the automatically extracted contours were measured. Moreover, these measurement sessions were an occasion to evaluate the speed-up achieved by our software compared to fully manual and semi-automatic procedure using Osiris. 


\section{Results}

The contours automatically extracted using the DAC model fit very well the actual vessel lumen boundaries (fig. 2). This qualitative result agrees with the quantitative comparisons. Average absolute difference between the first expert's measurements of area and ATHER measurements, was equal to $4 \%$ for the entire set of data. It is to be compared with the variability of the expert-performed measurements. The experts' intra-observer variability respectively was $6,4 \%$ and $2,3 \%$. The high value for the first expert can be explained by a longer time interval between the two measurements and by the fact that they were done on different screens. The inter-observer variability was as large as $4 \%$. It was calculated using a formula similar to (7), where $\operatorname{Area}(i)_{A T H E R}$ was replaced by the average of the two values measured by one expert for the slice $i$. Similarly, Area $(i)_{\text {EXPERT }}$ in the numerator was replaced by the average of the two values measured by the other expert, while in the denominator it was replaced by the average of both experts' measurements. The variability of ATHER's measurements was not more than $1,2 \%$. When the images are of good quality, the automatic contour gives very good results and is very insensitive to the position of the initialization point. In poor quality images, the initialization point may have more importance.
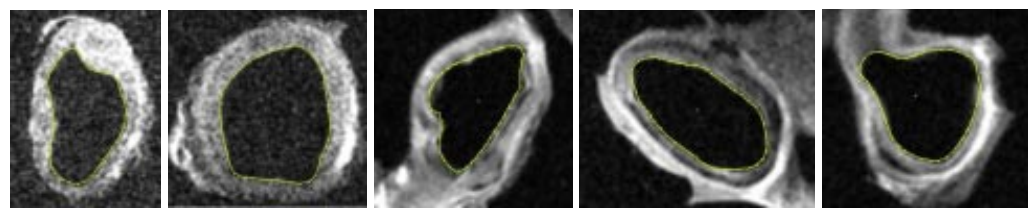

Fig. 2. Examples of results obtained with the DAC model in different territories. Note the variable quality (signal to noise ratio) of the images.

However, the maximum difference between ATHER and the first expert was as large as $19 \%$. Such large errors occurred in a few images of particularly poor quality (high level of noise) and in the case of images with residual quantities of formol in the vessel lumen. In the latter case, even an experienced human eye can hardly distinguish the boundary between these residues and the vessel wall. Hence the second expert judged these images not exploitable and they were not included in the subset of 45 slices for which the distance between the hand-made and automatically detected contours was also measured. For this subset, the maximum distance was less than 2 pixels (fig. 3) and the average distance varied from $1 / 3$ to $1 / 2$ pixel according to the spatial resolution of the images.

Lastly, let us underline the time savings obtained with ATHER. The expert used approximately 2 hours to obtain the measurements for 45 slices, with her usual operating mode. All the operations, from image file opening to $3 \mathrm{D}$ surface rendering of the contours automatically extracted by ATHER, took 30 seconds per series on the same PC. Adding visual inspection of each result and correction of the starting point in one series, all the data subset was analyzed within less than 15 minutes. 

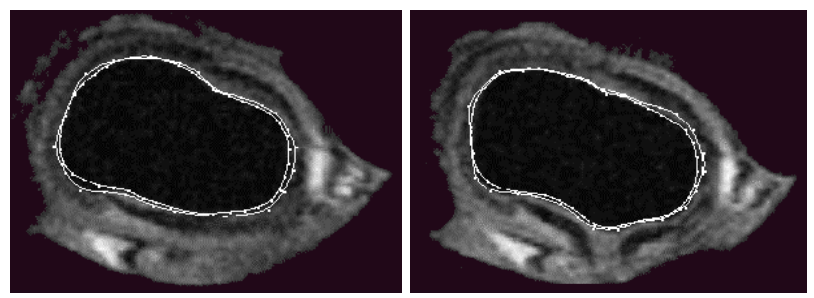

Fig. 3. Maximum distance between the contours drawn by ATHER and by the expert is less than 2 pixels. The curves' thickness, in this picture, is $1 / 3$ of the pixel size.

\section{Discussion and Conclusions}

The main characteristics of our active contour implementation are fast computation and results stability with different starting points within the vessel lumen. The latter characteristic reduces to minimum the user intervention. In particular, when propagating the boundary extraction from one slice to another, the previous contour's center can be used as starting point. This is very useful when slice spacing is large and the contour forms may vary significantly between neighboring slices. In our study the slice spacing is large so as to coincide with the thickness of histological slices. However, with smaller spacing, the current contour can be initialized by the previous contour, slightly reduced. In this case, the contour extraction is even faster and more robust.
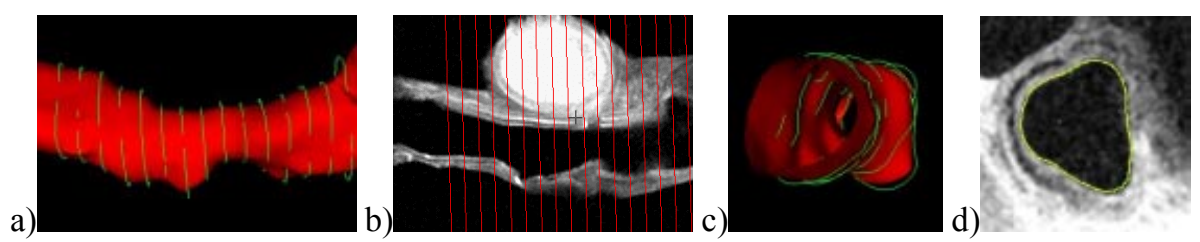

Fig. 4. 3D rendering of the internal surface of a vessel wall $(\mathrm{a}, \mathrm{c})$, generated from the extracted boundary points. Corresponding longitudinal (b) and cross-sectional 2D images (d).

The extracted planar contours are not only used for the purpose of quantification. They are also exploited for the sake of 3D visualization. From the detected boundary points, ATHER generates a shaded surface display (fig.4) which can be interactively animated. This is of great interest for visual assessment of the overall intraluminal morphology of the plaque.

The herein presented work is a part of a wider project. In the next task, ATHER will be applied to in vivo images from the same rabbits. This should give rise to a validation of our model also for the external boundaries of the vessels. In parallel, the same model was implemented in another graphical interface, named MARACAS, specially designed for the study of MR angiography images, i.e. images of the vascular lumen obtained after a contrast agent injection. It was already validated on 
images from phantoms [11]. Now, it is applied to images from our rabbits and from patients.

\section{Acknowledgements}

This work is supported by Rhône-Alpes region within AdeMo project. It is in the scope of the scientific topics of the GDR-PRC ISIS research group of the French National Center for Scientific Research (CNRS). The authors are grateful to Marcela Hernández-Hoyos and Eric Boix for their help in software implementation and to Emmanuelle Canet for her contribution in image acquisition and analysis.

\section{References}

1. Asdente M., et al. Evaluation of atherosclerotic lesions using NMR microimaging. Atherosclerosis, 1990. 80(3): 243-53

2. Yuan C., et al. Techniques for high-resolution MR imaging of atherosclerotic plaque. $J$ Magn Reson Imaging, 1994. 4(1): 43-49.

3. Toussaint J.F., et al. Magnetic resonance images lipid, fibrous, calcified, hemorrhagic, and thrombotic components of human atherosclerosis in vivo. Circulation, 1996. 94(5): 932-338.

4. Serfaty J.M., Chaabane L., et al. The value of T2-Weighted High Spatial Resolution Magnetic Resonance Imaging in Classifying and Characterizing Atherosclerotic Plaques: An In Vitro Study. Radiology, 2001, in press.

5. Chaabane L, Canet E, Serfaty JM, et al. Microimaging of atherosclerotic plaque in animal models. Magnetic Resonance Materials in Physics, Biology and Medicine 2000. 11: 58-60.

6. Kang X.J., et al. Analysis of the measurement precision of arterial lumen and wall areas using high resolution magnetic resonance imaging, MRM, 44:968-972, 2000.

7. Wink, O., et al. Semi-automated quantification and segmentation of abdominal aorta aneurysins from CTA volumes. in CARS Paris (France) 1999. 208-212.

8. Mc Inerney, T. and Terzopoulos D., Deformable models in medical image analysis : a survey. Med. Image Analysis, 1996. 1(2): 91-108.

9. Rueckert, D., et al. Automatic tracking of the aorta in cardiovascular MR images using deformable models. IEEE Trans. Med. Imaging, 1997. 16(5): 581-590

10. M. Kass, A. Witkin, D. Terzopoulos, Active contour models, Int J Comp Vision, 1988. 1: 321-331.

11. Hernández-Hoyos M., et al. A Deformable Vessel Model with Single Point Initialization for Segmentation, Quantification and Visualization of Blood Vessels in 3D MRA. MICCAI, Pittsburgh, Oct. 9-13, 2000. 735-745.

12. Desbleds-Mansard C. et al. Dynamic Active Contour Model for Size Independent Blood Vessel Lumen Segmentation and Quantification in High-Resolution Magnetic Resonance Images. Accepted for communication in Int. IAPR Conf. CAIP 2001, Warsaw (PL), Sept. 2001.

13. L. D. Cohen, On Active Contour Models and Balloons, Computer Vision, Graphics and Image Processing: Image Understanding 1991. 53(2): 211-218. 